\title{
Shave Excision
}

National Cancer Institute

\section{Source}

National Cancer Institute. Shave Excision. NCI Thesaurus. Code C51885.

A surgical procedure where thin slices are remove with or as if with a plane. 